\title{
Editorial
}

\section{Acknowledgement to Reviewers of Social Sciences in 2014}

Social Sciences Editorial Office, MDPI AG, Klybeckstrasse 64, CH-4057 Basel, Switzerland

Published: 8 January 2015

The editors of Social Sciences would like to express their sincere gratitude to the following reviewers for assessing manuscripts in 2014:

Anderson, Gwendolyn D.

Arruabarrena, Ignacia

Barner, John

Bernard, Claudia

Bernard, Kristin

Berthold, Anne

Beunen, Raoul

Bjørnskov, Christian

Bonneau, Claudine

Bowen, William M.

Britner, Preston A.

Burke, Jessica L.

Bywaters, Paul

Cameron, Gary

Carter, Michael J.

Chandler, Susan Meyers

Chen, Baiyun

Chiu, Yu-Ling

Christmann, Gabriela

Chua, Alton Y. K.

Claeys, Peter

Clapton, Gary

Clark, C. Brendan

Cohen, Mark A.

Connoly, Marie

Cornelius-White, Jeffrey $\mathrm{H}$.

Cossins, Anne

Cox, Michael

Coyne, Imelda

Cree, Viviene
Cross, Theodore P.

Curley, Melissa

Daedlow, Katrin

Davies, Kristin

Davies, Tammy

Day, Angelique

de Paúl, Joaquín

De Stefano, Lucia

Death, Jodi

Devaney, John

Dowdall, Clare

Drake, Brett

Elliot, Andrew

Elmore, Kristen C.

Ernstson, Henrik

Esposito, Tonino

Everson, Mark D.

Fallon, Barbara

Ferenczi, Nelli

Fergusson, David M.

Fiori, Katherine L.

Fluke, John D.

Garratt, Dean

Gazan, Rich

Gilbert, Ruth

Gulliver, Pauline

Hales, Andy

Harkins, Leigh

Harris, Patrick J.

Haslam, Alex
Hassink, Robert

Haynie, Denise

Hegger, Dries

Hodes, Deborah

Holgersen, Stale

Huang, Chien-Chung

Hudson, Malcolm

Hughes, Judy

Hurt, Stephen R.

Ibert, O.

Inoue, Takeshi

James, Helen

Jimoyiannis, Athanassios

Johnson, H. Durell

Jonson-Reid, Melissa

Jovanovic, Miroslav N.

Jugert, Philipp

Karatekin, Canan

Kauff, Mathias

Keddell, Emily

Kern, Leslie

Kerpelman, Jennifer

Kesner, John

Kilper, Heiderose

Kim, Sujin

Kirsten, Stalker

Knight, Kendra

Kollias, Christos

Kotakorpi, Kaisa

Lanier, Paul 
Lewicki, Aleksandra

Linderkamp, Tim

Ling, Minhua

Littlechild, Brian

Long, Maureen

Lonne, Bob

Luchok, Kathryn J.

Luke, Allan

Luong, Gloria

Margerison-Zilko, Claire

Martinez-Torteya, Cecilia

Masson, Judith

May-Chahal, Corinne

McArthur, Morag

McKenzie, Brad

McLaughlin, Hugh

McLoughlin, Catherine

McNamara, Patricia

Medvene, Louis J.

Meegan, Richard

Mendelson, Adam

Mendes, Philip

Montserrat Boada, Maria Carme

Morrison, Bruce

Navarro, Vicente

Nelson, Geoff

Nicholson, Jan

Nicholson, Joanne

Nordenfors, Monica
Nordtveit, Bjorn Harald

O'Donnell, Melissa

Oxley, Jennifer

Parent, Sylvie

Passini, Stefano

Pedersen, Morten

Petrie, S.

Pickett, Steward

Pithouse, Andrew

Prichard, Jeremy

Quayle, Ethel

Radford, Lorraine

Ratliffe, Katherine T.

Rennie, Frank

Riggio, Heidi R.

Runtz, Marsha

Russell, Kate

Sandberg, Linda

Sanders, Jackie

Sanders, Matt

Sapountzis, Antonis

Savona, Roberto

Savoy, Sarah

Scheibe, Cyndy

Scior, Katrina

Shanley, Jenelle

Sigfusdottir, Inga Dora

Skarbek, David

Skivenes, Marit
Sloth-Nielsen, Julia

Smith, Jordan

Smith, Mark

Spiwoks, Markus

Stokes, Jackie

Sultan, Dawood

Swenson, Cynthia Cupit

Taff, Derrick

Takagi, Daisuke

Tanaka-Ellis, Nobue

Taplin, Stephanie

Thai, Yvonne

Thomas, Nigel

Tonmyr, Lil

Topitzes, Dimitri

Tunstill, Jane

Van Holen, Frank

Walton, Gregory M.

Wang, Feihong

Wang, Renfeng

Watson, Alan

Welsh, Marc

Wilson, Michael L.

Winter, Aaron

Woods, Kevin

Wurtele, Sandy K.

$\mathrm{Yu}$, Yongking

Zimmermann, Lynn

(C) 2015 by the authors; licensee MDPI, Basel, Switzerland. This article is an open access article distributed under the terms and conditions of the Creative Commons Attribution license (http://creativecommons.org/licenses/by/4.0/). 